\title{
Características clínicas y demográficas en pacientes con cáncer de mama triple negativos, clínica Oncocare, 2004-2012
}

\section{Pedro Ramos}

Oncocare.

Objetivo. Describir las características clínicas y demográficas de las pacientes con cáncer de mama estado triple negativo.

Métodos. Información de clínica Oncocare en el período comprendido entre el $1^{\circ}$ de enero de 2004 hasta el 31 de mayo de 2012. Se incluyeron todas las pacientes con diagnóstico de cáncer de mama triple negativo (estadio I a IIIC). Se hicieron descripciones de las características clínicas y demográficas con estadística descriptiva.

Resultados. Un total de 33 pacientes fueron identificadas en el estudio. La edad media fue de 50 años, el tiempo de seguimiento promedio fue de 27,4 meses, se presentaron 8 muertes para una frecuencia del $24,4 \%$ y 13 recaídas que corresponden a un total del $39,9 \%$.

La presentación clínica fue estadio I (3,03\%), IIA (15,15\%), IIB $(24,24 \%)$, IIIA (12,12\%), IIIB (18,18\%), IIIC (27,27\%), siendo la mayoría estadios avanzados. El estado ganglionar se distribuyó en ganglios positivos en el $48 \%$ de los casos; 1 a 3 ganglios en el $8 \%$, 4 a 9 ganglios en el $20 \%$ y 10 o más ganglios en el $20 \%$. La mayoría de las pacientes pertenecen al régimen subsidiado $(69,70 \%)$; la mayoría de las pacientes eran grado histológico alto (3). Las pacientes recibieron quimioterapia neoadyuvante en el $81,82 \%$ y los esquemas más frecuentes fueron ACT $(48,15 \%)$ y AC $(33,33 \%)$; la respuesta a la quimioterapia neoadyuvante fue: respuesta objetiva en un $59,26 \%$, respuesta completa en un $18,52 \%$, respuesta parcial en un $40,74 \%$. Se presentaron recaídas en 12 casos para un $36,36 \%$ (sistémicas en 11 casos).

Conclusión. Las pacientes con cáncer de mama triple negativo son de alto riesgo de recaída y muerte, como se observa en esta serie de casos, lo cual coincide con lo reportado en la literatura.

\section{Experiencia con el protocolo de quimioterapia Rizzoli en pacientes con osteosarcoma de extremidad de alto grado, entre 2007 y 2010}

\author{
Natalia Arango
}

Objetivos. Describir las características clínicas y epidemiológicas de los pacientes con osteosarcoma de extremidad de alto grado tratados con los protocolos Rizzoli 2 o 4 en el Instituto Nacional de Cancerología entre enero de 2007 y diciembre de 2010, y contrastar con lo reportado en la literatura.

Materiales y métodos. Estudio descriptivo observacional. Se analizaron los registros de los pacientes con osteosarcoma de extremidad de alto grado tratados en el Instituto Nacional de Cancerología entre 2007 y 2010 con los protocolos Rizzoli 2 (4 pacientes) o Rizzoli 4 (14 pacientes), por medio de un instrumento de recolección de datos. La información obtenida se manejó con la base de datos Access.

Resultados. Se revisaron 18 registros de pacientes que habían recibido protocolo Rizzoli. La edad promedio fue 25 años, con mayor afectación del fémur. Luego de terapia neoayuvante, 15 de los 18 pacientes incluidos fueron llevados a procedimiento quirúrgico; el $60 \%$ logró preservación de la extremidad (9 pacientes); los 6 restantes requirieron amputación.
De los pacientes llevados a procedimiento quirúrgico, 10 tuvieron una mala respuesta neoadyuvante (tasa de viabilidad tumoral mayor del $10 \%)$.

Después de un seguimiento de dos años, el $57 \%$ de los pacientes se encontraban vivos.

Los eventos adversos más frecuentes no hematológicos corresponden a elevación de transaminasas grado 3, presentada durante todo el tratamiento. Las toxicidades hematológicas predominan en adyuvancia con leucopenia grado 2 y neutropenia grado 3 .

Conclusiones. Los pacientes adultos con osteosarcoma de extremidad de alto grado tratados con el protocolo Rizzoli 2 o Rizzoli 4 en el Instituto Nacional de Cancerología entre enero de 2007 y diciembre de 2010 presentaron tasas de respuesta, preservación de la extremidad y supervivencia menores que las reportadas en las publicaciones originales. 